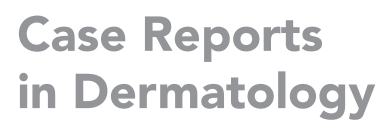

\title{
Sirolimus for the Management of Verrucous Venous Malformation: A Case Report and Literature Review
}

\author{
Ibrahim Al-Furaih $^{a} \quad$ Alanoud Al-Marzoug ${ }^{b} \quad$ Nada Al-Qadric \\ Saad Al-Ajlan ${ }^{\text {d }}$ \\ ${ }^{a}$ Department of dermatology, King Fahad Medical City, Riyadh, Saudi Arabia; bepartment \\ of dermatology, King Abdulaziz Medical City, Riyadh, Saudi Arabia; 'Primary health care \\ department, Ministry of Health, Riyadh, Saudi Arabia; ${ }^{\mathrm{d} D e p a r t m e n t}$ of dermatology, Alfaisal \\ University, King Faisal Specialist Hospital \& Research, Riyadh, Saudi Arabia
}

\section{Keywords}

Sirolimus · Verrucous venous malformation - Verrucous hemangioma · Case report

\begin{abstract}
Verrucous venous malformation (VVM) is a vascular malformation that is rarely reported in the literature which has vascular and hyperkeratotic components affecting mainly the extremities, difficult to treat, and often associated with complications mainly bleeding, ulceration with secondary infections, and limitation of movement. In this article, we report a successful improvement of VVM with sirolimus.
\end{abstract}

\section{Introduction}

Verrucous venous malformation (VVM), or verrucous hemangioma, is a rare vascular malformation composed of capillaries and veins in the dermis and subcutaneous tissue. It typically presents at birth or during early childhood with nonkeratotic, soft, violaceous macules, patches, and plaques that gradually become hyperkeratotic and verrucous over time. Although VVM most commonly affects the extremities, truncal and axillary involvement have been previously reported $[1,2]$.

VVM is generally difficult to treat [3]. Currently, combined therapy with laser (pulsed dye laser or ablative lasers) and surgery is considered the first-line of therapy [4]. Other treatment options include topical steroids, cryoablation, and radiotherapy with variable success [2]. Studies have shown that VVM is associated with a somatic mutation in the mitogen-activated

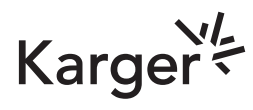




\section{Case Reports in Dermatology}

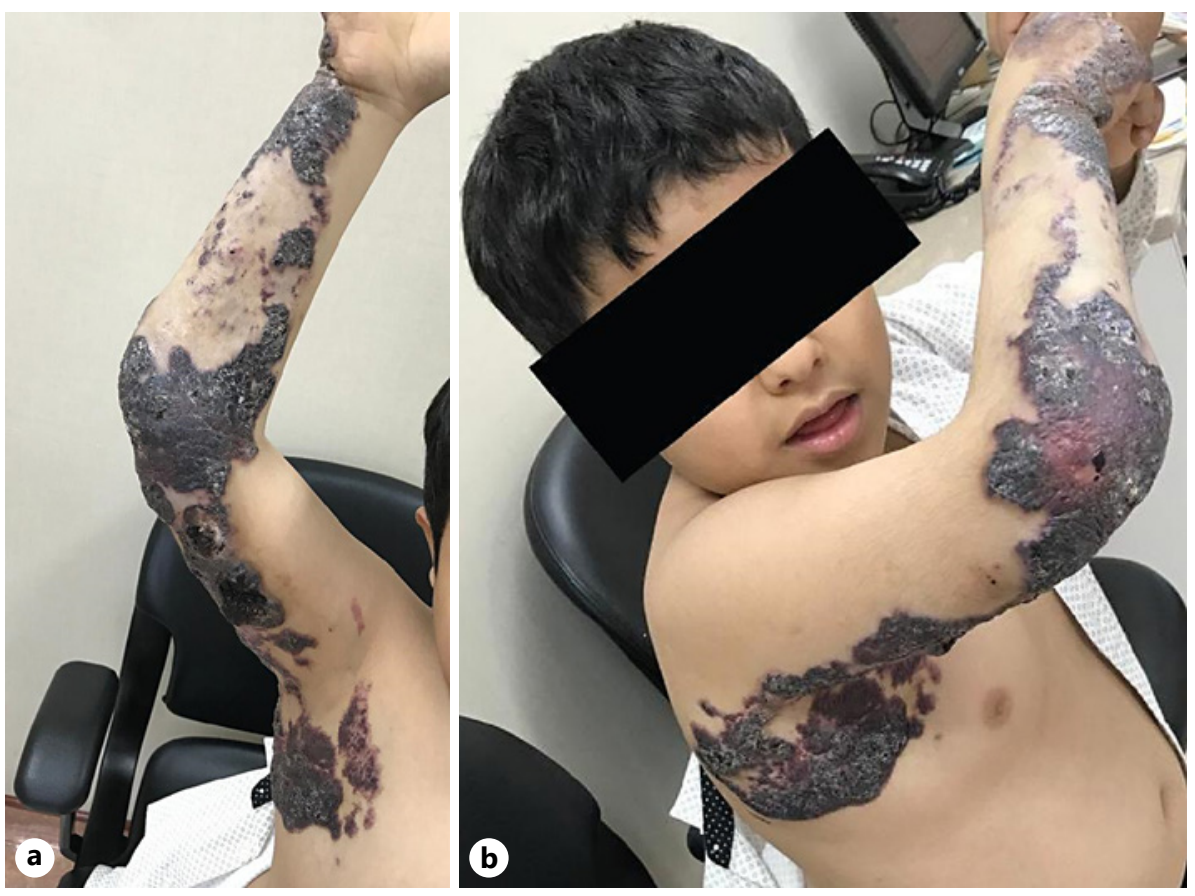

Fig. 1. a, b VVM upon initial presentation. The lesions are linear, verrucous, deeply red plaques with erosions and ulcerations. VVM, verrucous venous malformation.

protein kinase kinase kinase 3 gene (MAP3K3), which is an upstream molecule of the mammalian target of rapamycin signaling pathway [3,5]. Sirolimus, an inhibitor of mammalian target of rapamycin, has been increasingly used in the management of complicated vascular anomalies due to its results $[3,6]$. Herein, we present the successful use of sirolimus in the treatment of a patient with VVM.

\section{Case Report}

A 13-year-old boy, otherwise healthy, presented to the dermatology clinic 3 years ago with well-defined lesions over his right upper extremity, the left side of the abdominal wall, and the left gluteus. These lesions were noticed at birth and expanded in size relative to his growth. The lesions were associated with multiple episodes of bleeding, oozing, ulceration, and secondary impetiginization that required multiple hospital admission and intravenous antibiotics. The patient also reported a limited range of motion in his right upper extremity, mainly of the right elbow joint. The patient also expressed a cosmetic concern as well as the other symptoms mentioned. No similar condition was reported in the family. Moreover, no topical or systemic medication was used before, and there were no surgical interventions.

A clinical examination of the lesion showed a linear, verrucous, hyperkeratotic plaque extending from the radial aspect of the right hand and dorsal forearm up to the arm and axilla. There were also multiple well-defined, hyperpigmented brownish patches and plaques over the left side of the abdomen and left gluteus (shown in Fig. 1a, b).

On his initial clinic visit 3 years ago, an MRI of the right upper extremity was performed. The images showed multiple dilated, superficial, and deep tortuous structures involving the medial and posterior aspects of the right arm, mostly focused around

\section{Karger'}


Fig. 2. Appearance of VVM after treatment with sirolimus. There are now hyperkeratotic plaques that are nonnucleating or bleeding. VVM, verrucous venous malformation.

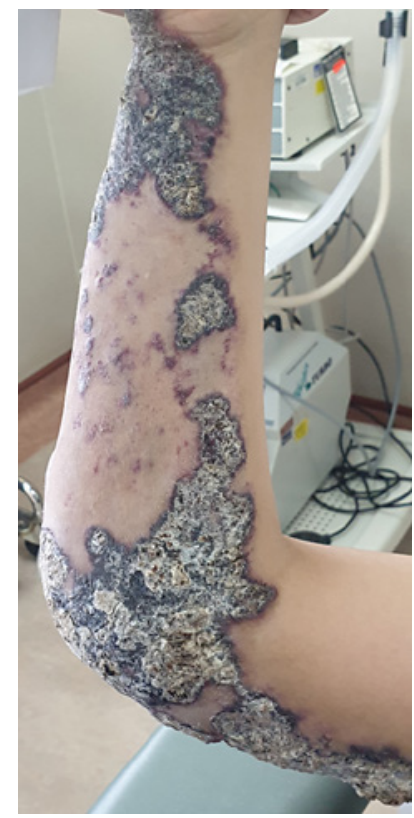

the posterior aspect of the right elbow and the medial and posterior aspects of the right forearm down to the right hand with the involvement of the skin and subcutaneous fat and the sparing of muscle.

A diagnosis of VVM was made on the basis of clinical, histopathological, and radiological findings. The patient was started on sirolimus at a total dose of $1.6 \mathrm{mg} / \mathrm{m}^{2}$ per day, divided into 2 doses per day for 3 years. Baseline investigations prior to therapy including complete blood count, liver and renal function tests, urinalysis, and hepatitis B and C panel were all within normal ranges.

The patient was followed up every 3 months during the first year and then every 6 months thereafter. The target serum sirolimus level was between 6 and $15 \mathrm{ng} / \mathrm{mL}$. The weight and serum levels were measured at every visit, and the dosage was adjusted accordingly. After 3 years of sirolimus therapy, the patient and his parents reported a notable recovery in bleeding, ulceration, and infection episodes. The patient also reported a marked improvement in his right elbow joint mobility (shown in Fig. 2). No side effects were noted by the physician or reported by our patient.

\section{Discussion}

The International Society for the Study of Vascular Anomalies scheme classifies vascular anomalies into vascular malformations and vascular tumors. Verrucous hemangioma is a misnomer and has been classified as a rare, slow flow venous malformation that usually presents at birth or during early childhood [1].

This specific entity is frequently misdiagnosed and under-recognized; therefore, it should be considered as a differential diagnosis for similar skin lesions and must be differentiated from infantile hemangioma, verrucous epidermal nevus, angiokeratoma, and lymphatic and other venous malformations. The typical clinical presentation of verrucous hemangioma is red-brown, hyperkeratotic plaques or nodules that may show linear or blaschkoid-like arrangements. They are most commonly localized on the extremities and buttocks and less commonly on the trunk and axilla. 
The lesions typically have a vascular and hyperkeratotic component; the vascular component is typically present at birth as a red, violaceous stain, whereas the hyperkeratotic component develops with age [2]. Variable degrees of hyperkeratosis are seen that may lead to pain, bleeding, ulceration, and secondary infections as was observed in this patient. This patient's lesions showed a linear configuration, which is consistent with what has been reported in the literature, as shown in Table $1[1,2]$. It has been suggested that lesions with linear arrangements may reflect genetic mosaicism [7].

The treatment of vascular malformations is complex and requires a multidisciplinary team. Both medical and surgical interventions are often needed. Recurrence after surgical excision has been reported in the past.

Recently, there is growing evidence that sirolimus can be used in the management of complicated vascular anomalies due to its antiproliferative and antiangiogenic properties, with promising results [6]. In our report, neither the surgical option (due to body surface area involvement and risk of recurrence) or laser options (as he was living outside the city) is feasible options. A retrospective analysis of 10 patients with VVM at a mean age of 17 months who were treated with oral sirolimus was conducted; they reported marked improvement of symptoms with volume reduction of lesions of $>90 \%$, suggesting that sirolimus is a safe, reliable, and effective treatment [3]. In this case, oral sirolimus administered at a dose of 1.6 $\mathrm{mg} / \mathrm{m}^{2}$ divided into a twice daily dosing led to a volume reduction of the lesions, absence of bleeding, ulceration, secondary infections, and most significantly, an improvement in the right elbow joint's range of motion.

Unfortunately, the age which is most responsive to sirolimus and whether there is a risk of recurrence after discontinuation are unknown. We will continue sirolimus as long as the patient is feeling improvement and not limited by the side effect profile. In the future, we might shift/combine it with laser options (pulsed dye or ablative laser) if its applicable with our patient.

\section{Conclusion}

VVM is a disfiguring disease, which in severe cases can lead to contractures, which affect daily life activities. Currently, laser and surgical methods are the first-line treatment options. This report supports sirolimus as an interesting option for treatment.

\section{Statement of Ethics}

This report complies with the Declaration of Helsinki ethical principles. The ethical approval was acquired for this case report, and written informed consent was obtained from the patient for publication of this case report and any accompanying images.

\section{Conflict of Interest Statement}

The authors have no conflicts of interest to declare.

\section{Funding Sources}

No funding sources are involved in the study.

\section{Karger'}


Case Reports

in Dermatology
Case Rep Dermatol 2021;13:298-303 DOI: 10.1159/000515984

(c) 2021 The Author(s). Published by S. Karger AG, Basel www.karger.com/cde

Al-Furaih et al.: Sirolimus for Verrucous Venous Malformation
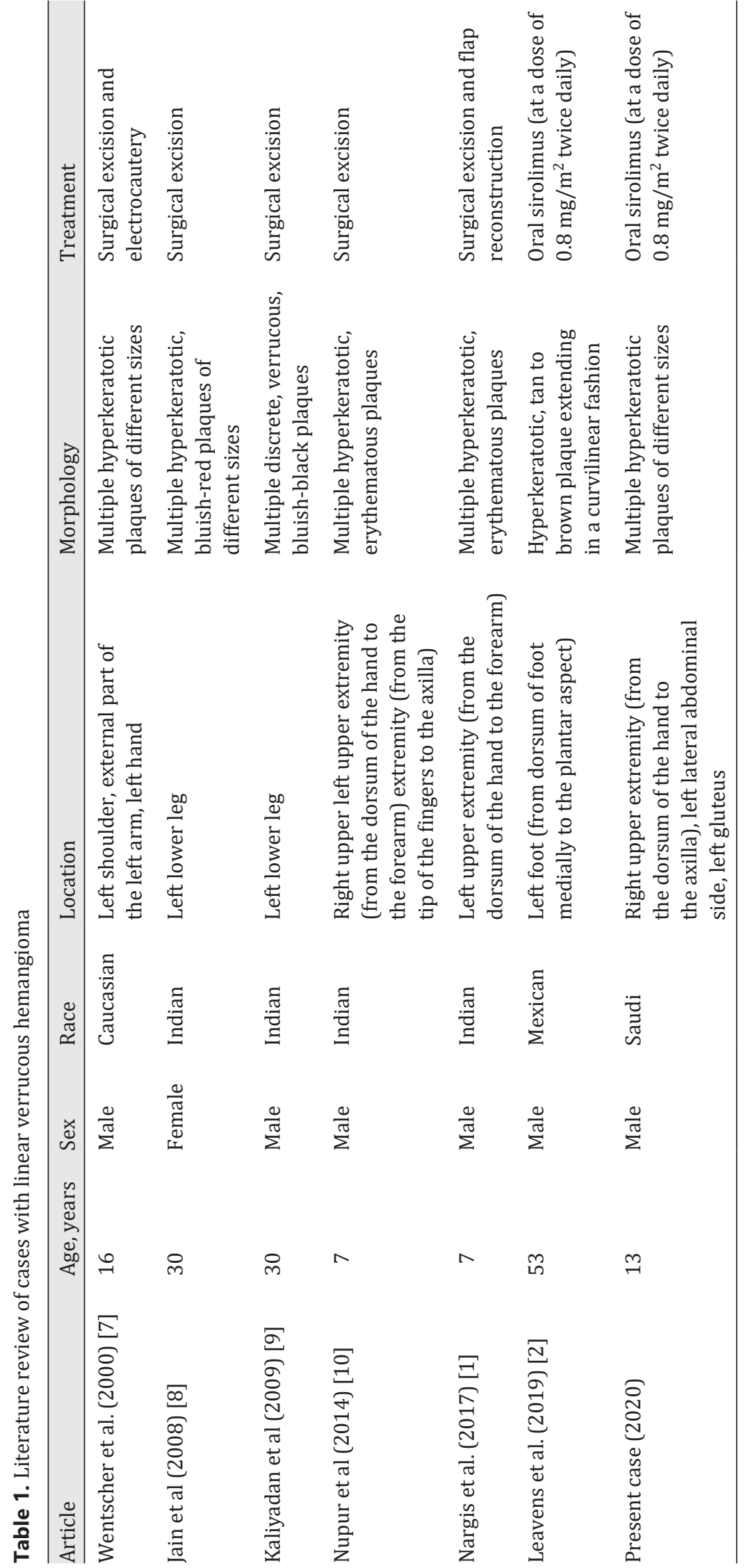


\section{Case Reports in Dermatology}

\begin{tabular}{l|l}
\hline Case Rep Dermatol 2021;13:298-303 \\
\hline DOI: 10.1159/000515984 & $\begin{array}{l}\text { @ 2021 The Author(s). Published by S. Karger AG, Basel } \\
\text { www.karger.com/cde }\end{array}$ \\
\hline
\end{tabular}

Al-Furaih et al.: Sirolimus for Verrucous Venous Malformation

\section{Author Contributions}

Ibrahim Alfuraih: conceptualization, data collection, and manuscript writing. Alanoud Al-Marzoug: data collection and manuscript writing. Nada Al-Qadri: manuscript writing and revision. Saad Al-Ajlan: conceptualization and revision.

\section{References}

1 Nargis T, Pinto M, Bhat S, Shenoy MM. Linear verrucous hemangioma of the upper limb: a rare case. Dermatol Online J. 2017 Jun;23(6):1-3.

2 Leavens J, Worswick S, Kim GH. Verrucous venous malformation. Dermatol Online J. 2019 Dec;25(12):1-4.

3 Zhang G, Chen H, Zhen Z, Chen J, Zhang S, Qin Q, et al . Sirolimus for treatment of verrucous venous malformation: a retrospective cohort study. J Am Acad Dermatol. 2018 Jul 23;80(2):556-8.

4 Oppermann K, Boff AL, Bonamigo RR. Verrucous hemangioma and histopathological differential diagnosis with angiokeratoma circumscriptum neviforme. An Bras Dermatol. 2018 Oct;93(5):712-5.

5 Couto JA, Vivero MP, Kozakewich HP, Taghinia AH, Mulliken JB, Warman ML, et al. A somatic MAP3K3 mutation is associated with verrucous venous malformation. Am J Hum Genet. 2015 Mar 5;96(3):480-6.

6 Freixo C, Ferreira V, Martins J, Almeida R, Caldeira D, Rosa M, et al Efficacy and safety of sirolimus in the treatment of vascular anomalies: a systematic review. J Vasc Surg. 2020 Jan;71(1):318-27.

7 Wentscher U, Happle R. Linear verrucous hemangioma. J Am Acad Dermatol. 2000 Mar 1;42(3):516-8.

8 Jain VK, Aggarwal K, Jain S. Linear verrucous hemangioma on the leg. Indian J Dermatol Venereol Leprol. 2008; 74(6):656-8.

9 Kaliyadan F, Dharmaratnam AD, Jayasree MG, Sreekanth G. Linear verrucous hemangioma. Dermatol Online J. 2009 May 15;15(5):15. 19624993.

10 Nupur P, Savant SS, Kumar P, Hassan S. Linear verrucous hemangioma. Indian Dermatol Online J. 2014;5(6): 136-7. 